\title{
An Anomalous Hammett Correlation for a Series of Substituted 3-Benzyl-2-phenyl-1,3-thiazolidin-4-ones
}

\author{
Daniel McGarity ${ }^{1}$, John Tierney ${ }^{1} \&$ Anthony Lagalante ${ }^{2}$ \\ ${ }^{1}$ Department of Chemistry, Pennsylvania State University, Brandywine Campus, Media, PA, USA \\ ${ }^{2}$ Villanova University, Villanova, PA, USA \\ Correspondence: John Tierney, Department of Chemistry, Pennsylvania State University, Brandywine Campus, \\ 25 Yearsley Mill Road, Media, PA 19063, USA. E-mail: jxt4@psu.edu
}

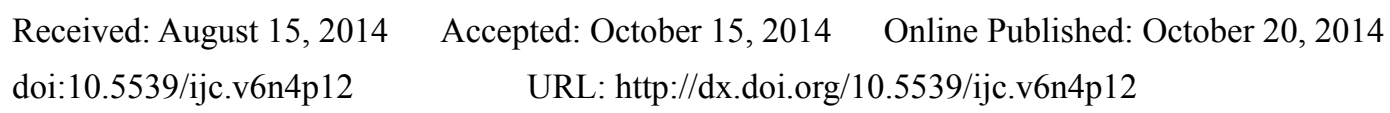

\begin{abstract}
A series of 3-benzyl-2-phenyl-1,3-thiazolidin-4-one derivatives, with substituents at the N-benzyl site, was synthesized and characterized. Excluding the $p-\mathrm{MeO}$ and $m-\mathrm{Br}$ derivatives, distinct correlations between Hammett constant $\sigma$ and ${ }^{13} \mathrm{C}$ substituent chemical shifts were observed in the $\mathrm{C} 2, \mathrm{C} 4$, and $\mathrm{C} 5$ carbons in the thiazolidin-4-one ring. This was unexpected because the transmission of substituent effects appears to be occurring via the $\mathrm{sp}^{3}$ hybridized N-benzyl carbon. No discernible correlation was seen for the N-benzyl carbon; the carbon through which the effects were occurring. Correlations for substituent chemical shifts to Swain Lupton substituent parameters $r$ and $f$ constants were also attempted. Similar to the Hammett correlations, C2, $\mathrm{C} 4$, and C5 showed a reasonable degree of correlation with minimal to no improvement over Hammett constants; similarly, as with the Hammett correlations, the benzyl carbon exhibited no correlation with Swain Lupton parameters.
\end{abstract}

Keywords: Thiazolidin-4-ones, ${ }^{1} \mathrm{H}$ NMR, ${ }^{13} \mathrm{C}$ NMR, substituent effects

\section{Introduction}

A number of thiazolidinones systems have been investigated for their diverse biological activities, including potential anti-tumor, anti-inflammatory and anti-HIV agents (Hafez \& El-Gazzar, 2009; Vigorita et al., 2001; Ravichandran, 2009). A growing series of thiazolidinone systems have been studied (Figure 1), and these studies have been previously been described (Cannon et al., 2013). ${ }^{1} \mathrm{H}$ NMR data was used to determine how phenyl or benzyl ring substituents for diaryl thiazolidinones (Series 1-4) affected electron density at the C-2 proton $\left(\mathrm{H}_{\mathrm{x}}\right)$ and diastereotopic protons at $\mathrm{C}-5\left(\mathrm{H}_{\mathrm{a}}\right.$ and $\left.\mathrm{H}_{\mathrm{b}}\right)$.

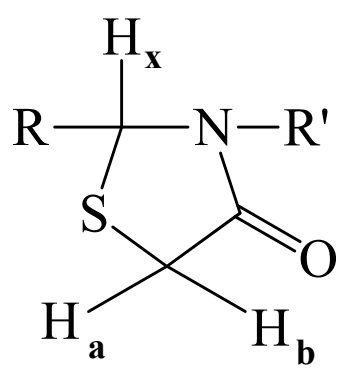

\footnotetext{
Series 1: $\mathbf{R}=$ phenyl, $\mathbf{R}^{\prime}=m$ - and $p$-substituted phenyl

Series 2: $R=m$ - and $p$-substituted phenyl, $R^{\prime}=$ phenyl

Series 3: $\mathbf{R}=\mathbf{R}^{\prime}=\boldsymbol{m}$ - and $\boldsymbol{p}$-substituted phenyl

Series 4: $R=m$ - and $p$-substituted phenyl, $R^{\prime}=$ benzyl

Series 5: $R=$ phenyl, $R^{\prime}=m$ - and $p$-substituted benzyl

Series 6: $R=m$ - and $p$-substituted phenyl, $R^{\prime}=2$-pyridyl

Series 7: $R=$ trichloromethyl, $\mathbf{R}^{\prime}=m$ - and $p$-substituted phenyl

Series 8: $R=m$ - and $p$-substituted phenyl, $R^{\prime}=$ cyclohexyl
}

Figure 1. All thiazolidinone studies published to this point. The meta and para substituents on phenyls designated by $\mathrm{R}$ and $\mathrm{R}^{\prime}$ are: $p$ - $\mathrm{NO}_{2} ; m-\mathrm{NO}_{2} ; p-\mathrm{F} ; m-\mathrm{F} ; p-\mathrm{Cl} ; m-\mathrm{Cl} ; p-\mathrm{Br} ; m-\mathrm{Br} ; \mathrm{H}, p-\mathrm{Me} ; m-\mathrm{Me} ; p-\mathrm{MeO} ; m-\mathrm{MeO}$

Thiazolidinone rings can have different conformations when solid and in solution (Figure 2). For 2,3-diaryl thiazolidinones (Series 1, 2 and 6), it was shown that conformer A was the preferred solid state confirmation, while conformer B was predominant in solution according to NMR observations in a number of similar systems 
(Yennawar et al., 2014). Similar results have been seen with the unsubstituted N-cyclohexyl compound from Series 8 and recently experiments show that the unsubstituted compound in Series 5 also exhibited A (Figure 2) as the preferred solid state conformation (Fun et al., 2011; Cannon et al., 2013).

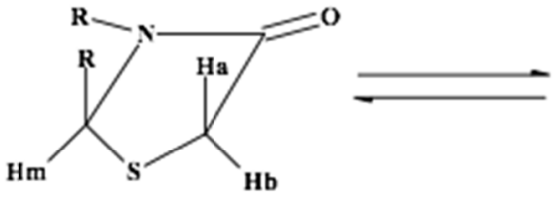

A

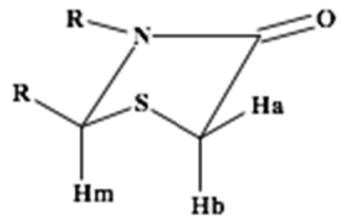

$\mathbf{B}$

Figure 2. Pseudo-axial and pseudo equatorial ring conformers

The ORTEP model in Figure 3 shows the crystal structure of the unsubstituted 3-benzyl-2-phenyl-1,3-thiazolidin-4-one. The phenyl group has the same orientation relative to the thiazolidinone-4-one ring as the phenyl group in Series 8 (Cannon et al., 2013). The plane of the benzene ring, however, is significantly twisted relative the N-benzyl group in Series 5; the benzyl group having much more freedom to rotate than in the other systems.

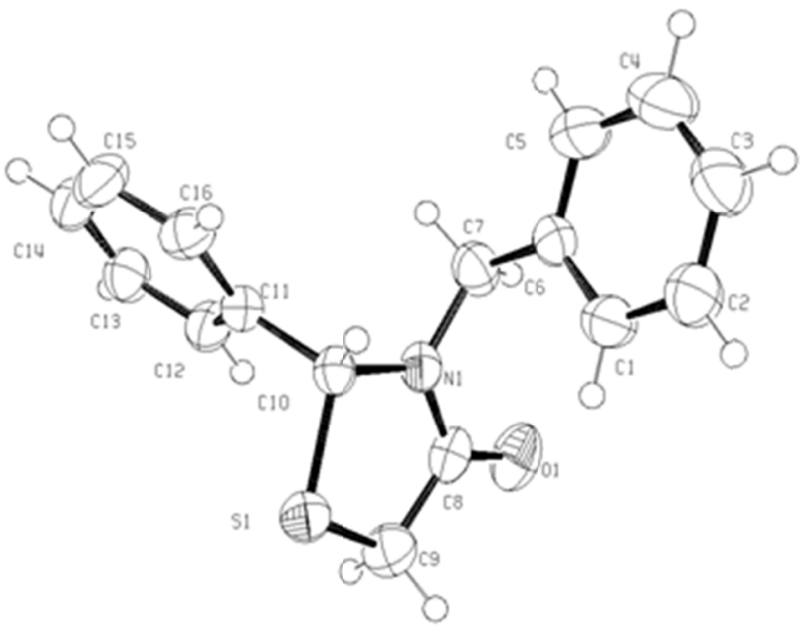

Figure 3. Ortep diagram 3-benzyl-1,3-thiazolidin-4-one. Note that the numeric assignments for atoms are inconsistent with IUPAC nomenclature

Previous work has illustrated that substituent Hammett $(\sigma)$ and Swain Lupton $r$ (resonance) and $f$ (inductive) correlations can indicate the degree of electron density at various sites in the thiazolidinone ring subject to substituents in the aryl moieties (Tierney et al., 1996a). These electron density correlations have been studied by measuring ${ }^{13} \mathrm{C}$ chemical shift data at $\mathrm{C} 2, \mathrm{C} 4$ and $\mathrm{C} 5$ for Series 1 through 6 (Figure 1), where substituents were attached to either a phenyl or benzyl sites at C2 and N3, respectively. Substituent chemical shifts (SCS) have previously shown a linear relationship with substituent Hammett constants (Silverberg et al., 2013; Tierney et al., 1996b). In this study, SCS was defined by the empirical relation SCS $=\log \left(\delta_{\mathrm{x}} / \delta_{\mathrm{h}}\right)$, where $\delta_{\mathrm{x}}$ represents the ${ }^{13} \mathrm{C}$ chemical shift for a substituted compound, and $\delta_{\mathrm{h}}$ is the chemical shift for the unsubstituted, control compound. SCS values for a series of substituted 3-benzyl-2-phenyl-1.3-thiazolidin-4-ones, (Series 4, Figure 1) were attempted using Hammett constants according to the relationship SCS $=\rho \sigma$. Previously, a limited investigation of a similar system (Series 5) with three substituents and the unsubstituted compound showed no correlation, and none was expected here. In addition, a substituent chemical shift correlation was also attempted with Swain Lupton dual substituent parameters by the equation $\mathrm{SCS}=r \mathrm{R}+f \mathrm{~F}$. The results of the study for this more extensively substituted Series 5 than the one previously reported follows (Tierney et al., 1996b). 


\section{Results and Discussion}

For the series under consideration, Series 5, substituent chemical shifts for all of the carbons in the thiazolidinone ring appear to exhibit a linear Hammett correlation as shown in (Figures 4-6), with the exception of the para-methoxy and meta-bromo groups. This is in agreement with similar work previously carried out for Series 1-3 shown in Figure 1 (Woolston et al., 1992; Tierney et al., 1996a). For Series 8, a Hammett correlation was observed for $\mathrm{C} 2$, but not $\mathrm{C} 4$ or $\mathrm{C} 5$ (Cannon et al., 2013) and the strongest Hammett correlations for all the other series has always been for the transmission of substituent effects to the $\mathrm{C} 2$ site in the thiazolidinone ring. As Figure 7 shows, the benzyl carbon did not appear to have any relationship with the benzyl substituent. Hammett correlations were strengthened when the $p$-methoxy and $m$ - $\mathrm{Br}$ derivatives were excluded (Table 1). Significant deviations with the para-methoxy group have been previously noted (Cannon et al., 2013). One proposed reason is that strong donating groups like para-methoxy may be responsible for altering the geometry in a way that reduces the degree of conjugation (Johnson, 1978). Although the reason for the deviation due to the $m$ - $\mathrm{Br}$ group is not yet clear, meta-substituted derivatives have been shown to be less reliable at predicting chemical shifts. In this instance the size of the meta-bromo group may be altering the geometry of the N-benzyl ring relative to all the other meta substituted compounds. The value of $\rho$ was negative for the $\mathrm{C} 2$ and $\mathrm{C} 4$ correlations, indicating that those sites favored a positive charge density. The trend for C5 showed a positive $\rho$ value, and therefore, it favors a negative charge density; this agrees with observations from previous work (Cannon et al., 2013).

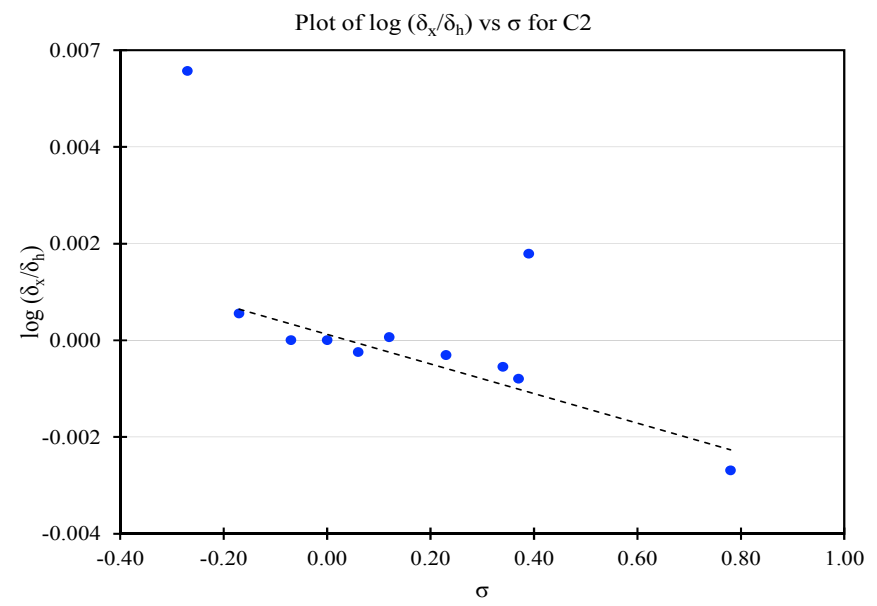

Figure 4. Plot of $\sigma$ constant $v s$ SCS for all ${ }^{13} \mathrm{C}$ shifts for the thiazolidin-4-one $\mathrm{C} 2$ carbon

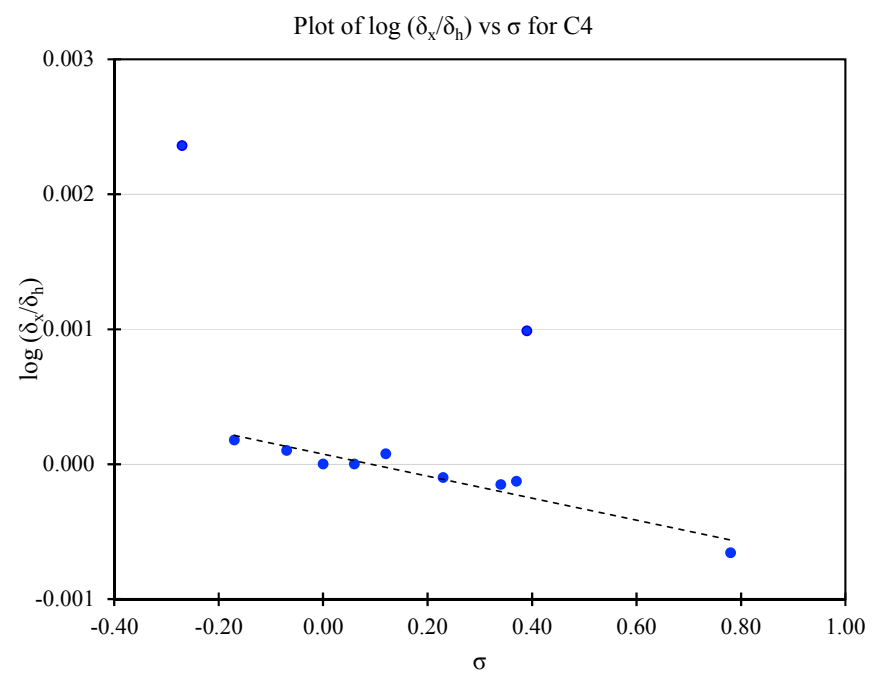

Figure 5. Plot of $\sigma v s \mathrm{SCS}$ for all ${ }^{13} \mathrm{C}$ shifts for the thiazolidin-4-one $\mathrm{C} 4$ carbon 


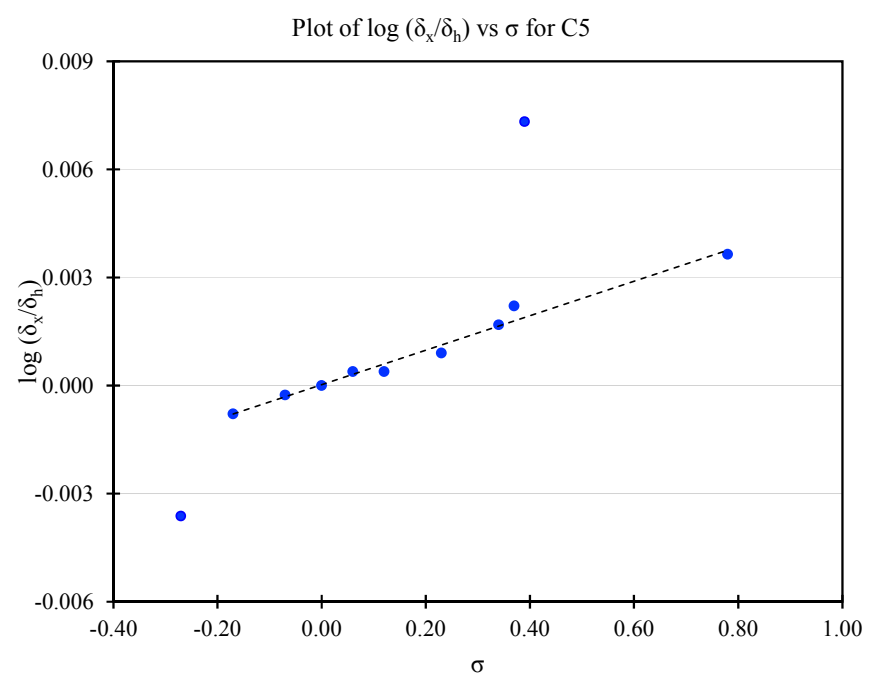

Figure 6. Plot of $\sigma v$ SCS for all ${ }^{13} \mathrm{C}$ shifts for the thiazolidin-4-one $\mathrm{C} 5$ carbon

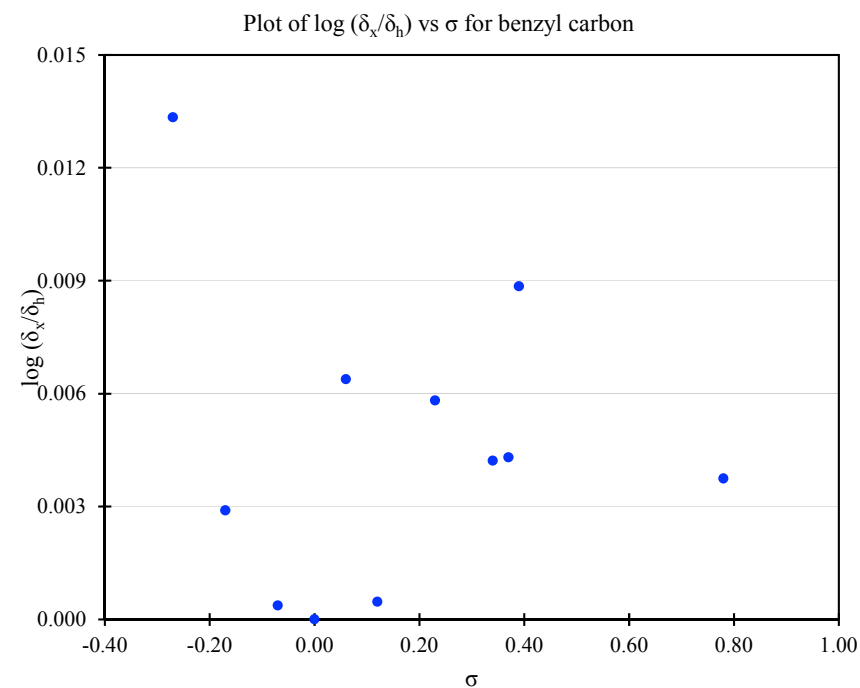

Figure 7. Plot of $\sigma$ vs SCS for all ${ }^{13} \mathrm{C}$ shifts for the N-benzyl carbon

Table 1. Equations for improved correlations between Hammett and Substituent Chemical Shifts when $m$-Br and $p$-methoxy derivatives are removed from consideration

\begin{tabular}{ccc}
\hline Carbon & Equation & $\mathbf{R}^{2}$ \\
\hline C2 & $\mathrm{y}=-0.0034 \mathrm{x}+1.0 \mathrm{E}-4$ & 0.8983 \\
$\mathrm{C} 4$ & $\mathrm{y}=0.0008 \mathrm{x}+7.42 \mathrm{E}-5$ & 0.9154 \\
$\mathrm{C} 5$ & $\mathrm{y}=0.0048 \mathrm{x}+2.86 \mathrm{E}-5$ & 0.9814 \\
Benzyl & $\mathrm{y}=0.0030 \mathrm{x}+2.60 \mathrm{E}-3$ & 0.1343 \\
\hline
\end{tabular}

The Swain Lupton correlations for C2, C4, and C5 are shown in Table 2 but there is no significant improvement in the correlations. As with the Hammett correlations, there was no noticeable correlation with ${ }^{13} \mathrm{C}$ shifts in the benzyl carbon. Here, it was also noted that correlation improved if the data for the $p$ - $\mathrm{MeO}$ and $m$ - $\mathrm{Br}$ derivatives 
were not considered in the correlation.

Table 2. Swain Lupton parameters fitted to the equation $\mathrm{SCS}=r \mathrm{R}+f \mathrm{~F}$ when $m$ - $\mathrm{Br}$ and $p-\mathrm{MeO}$ were removed from consideration

\begin{tabular}{lccc}
\hline Carbon & $\boldsymbol{r}$ & $\boldsymbol{f}$ & $\mathrm{R}^{2}$ \\
\hline $\mathrm{C} 2$ & $-2.65 \mathrm{E}-03$ & $-3.44 \mathrm{E}-03$ & 0.8817 \\
$\mathrm{C} 4$ & $6.41 \mathrm{E}-04$ & $8.22 \mathrm{E}-04$ & 0.9202 \\
C5 & $2.35 \mathrm{E}-03$ & $5.11 \mathrm{E}-03$ & 0.8656 \\
Benzyl & $8.73 \mathrm{E}-04$ & $7.01 \mathrm{E}-03$ & 0.4809 \\
\hline
\end{tabular}

Most interestingly is the observed apparent lack of correlation of substituent effects at the N-benzyl site, particularly when this site emulates, in some respects the $\mathrm{C} 2$ site which has previously always shown relatively strong substituent correlations. Further, as previously mentioned, Hammett $\sigma$ correlations would not be predicted beyond the N-benzyl carbon because it is $\mathrm{sp}^{3}$ hybridized.

\section{Conclusion}

This work has quantified the effect of benzyl substituents on the ${ }^{13} \mathrm{C}$ shift for $\mathrm{C} 2, \mathrm{C} 4$, and $\mathrm{C} 5$ in the thiazolidinone ring for Series 5 (Figure 1). Excluding the $p$-methoxy and $m$-Br substituents, both Hammett and Swain Lupton constants exhibit a reasonable degree of correlation for the $\mathrm{C} 2, \mathrm{C} 4$ and $\mathrm{C}_{5}{ }^{13} \mathrm{C}$ shifts. The deviations of the $p$-methoxy derivative has been previously observed (Woolston et al., 1992; Tierney et al., 1996a), but this study appears to be the first in which the $m$-Br derivative shows a large deviation which might be attributable to steric effects due to its size in this particular system. There was no discernable substituent correlation for ${ }^{13} \mathrm{C}$ chemical shifts for the N-benzyl carbon yet correlations were observed at sites beyond this carbon. It is unusual that Hammett correlations would be observed at the sites indicated because the transmission of substituent effects requires a high degree of conjugation from substituent to site. This observation calls into question the degree of $\mathrm{sp}^{3}$ hybridization at the N-benzyl carbon, and the observation has caused us to initiate molecular modelling studies on this system.

\section{Experimental}

\subsection{General}

The target compounds were prepared using a previously described procedure (Surrey, 1947). A Mel-Temp capillary melting point apparatus was employed and all Melting points are uncorrected. NMR spectra were recorded on a Bruker 400 at $291 \mathrm{~K}$ using parameters that have been reported previously (Cannon, 2013). IR spectra were obtained using $\mathrm{NaCl}$ plates supplied by Janos Technology, Inc, the deposition of a thin film, by evaporation. A Nicolet Nexus 670 spectrometer was used and the conditions of operation have been previously reported (Cannon, 2013). An Applied Biosystems API 2000 Triple Quadrupole Mass Spectrometer was used to determine molecular masses implementing an Electrospray ionization technique. A $1 \%(\mathrm{v}: \mathrm{v})$ formic acid methanol mixture infused at $10 \mu \mathrm{L} / \mathrm{min}$ was used as the electrospray solution. Curtain gas, $20 \mathrm{~mL} / \mathrm{min}$; collision gas, $0 \mathrm{~mL} / \mathrm{min}$; ionspray potential, $5.5 \mathrm{kV}$; temperature, $298 \mathrm{~K}$; declustering potential, $60 \mathrm{~V}$; focusing potential, $200 \mathrm{~V}$; entrance potential, $10 \mathrm{~V}$; collision energy, 25 units; cell exit potential, $4 \mathrm{~V}$. Product yields are based on imines (as limiting reactant) produced from reaction of substituted benzyl-amines and benzaldehyde. Maximization of yields was not attempted. All correlations and statistics were obtained using Excel in Microsoft Office. ORTEP models were made with ORTEP version 2013.1 (Farrugia, 2012).

\subsection{Synthesis and Characterization of Compounds}

3-(p-nitrobenzyl)-2-phenyl-1,3-thiazolidin-4-one (5a) (2\%); m.p. 150-156 ${ }^{\circ} \mathrm{C} ; \mathrm{cm}^{-1} 1674(\mathrm{C}=\mathrm{O}) ;{ }^{1} \mathrm{H}$ NMR $\left(\mathrm{CDCl}_{3}\right)$ 8.18-7.23 (9H, m, aromatics), $5.42(1 \mathrm{H}, \mathrm{d}, \mathrm{C} 2, \mathrm{~J}=1.9 \mathrm{~Hz}), 5.08(1 \mathrm{H}, \mathrm{d}, \mathrm{J}=15.2 \mathrm{~Hz}), 3.93(1 \mathrm{H}, \mathrm{dd}, \mathrm{C} 5$, $\mathrm{J}=1.8 \mathrm{~Hz}$, and $15.8 \mathrm{~Hz}), 3.84(1 \mathrm{H}, \mathrm{d}, \mathrm{J}=15.1 \mathrm{~Hz}), 3.82(1 \mathrm{H}, \mathrm{d}, \mathrm{C} 5, \mathrm{~J}=15.7 \mathrm{~Hz}) ;{ }^{13} \mathrm{C}$ NMR: $171.92(\mathrm{C} 4)$, 147.98, 143.31, 138.76, 130.02, 129.68, 129.50, 127.70, 124.38, (ar), 63.57 (C2), 46.22, 33.18 (C5); (m/z) 315 $\left([\mathrm{M}+\mathrm{H}]^{+}\right) \mathrm{C}_{16} \mathrm{H}_{14} \mathrm{~N}_{2} \mathrm{O}_{3} \mathrm{~S}(314.36)$. 
3-(p-fluorobenzyl)-2-phenyl-1,3-thiazolidin-4-one (5b) (68\%); m.p. $152-153{ }^{\circ} \mathrm{C} ; \mathrm{cm}^{-1} 1668(\mathrm{C}=\mathrm{O}) ;{ }^{1} \mathrm{H}$ NMR $\left(\mathrm{CDCl}_{3}\right) 7.40-6.80(9 \mathrm{H}, \mathrm{m}$, aromatics), $5.39(1 \mathrm{H}, \mathrm{d}, \mathrm{C} 2, \mathrm{~J}=1.6 \mathrm{~Hz}), 5.07(1 \mathrm{H}, \mathrm{d}, \mathrm{J}=14.7 \mathrm{~Hz}), 3.90(1 \mathrm{H}, \mathrm{dd}, \mathrm{C} 5$, $\mathrm{J}=1.0 \mathrm{~Hz}$, and $15.8 \mathrm{~Hz}), 3.75(1 \mathrm{H}, \mathrm{d}, \mathrm{J}=15.6 \mathrm{~Hz}), 3.55(1 \mathrm{H}, \mathrm{d}, \mathrm{C} 5, \mathrm{~J}=14.7 \mathrm{~Hz}) ;{ }^{13} \mathrm{C}$ NMR: $171.66(\mathrm{C} 4)$, $139.34,131.59,131.55,130.67,130.59,129.73,129.58,127.60$ (ar), 63.17 (C2), 45.94, 33.43 (C5) ; (m/z) 288 $\left([\mathrm{M}+\mathrm{H}]^{+}\right) \mathrm{C}_{16} \mathrm{H}_{14} \mathrm{NOSF}(287.35)$.

3-(m-fluorobenzyl)-2-phenyl-1,3-thiazolidin-4-one (5c) (17\%); m.p. 106-107 ${ }^{\circ} \mathrm{C} ; \mathrm{cm}^{-1} 1674(\mathrm{C}=\mathrm{O}) ;{ }^{1} \mathrm{H}$ NMR $\left(\mathrm{CDCl}_{3}\right) 7.39-6.95(9 \mathrm{H}, \mathrm{m}$, aromatics), $5.39(1 \mathrm{H}, \mathrm{d}, \mathrm{C} 2, \mathrm{~J}=1.8 \mathrm{~Hz}), 5.09(1 \mathrm{H}, \mathrm{d}, \mathrm{J}=14.9 \mathrm{~Hz}), 3.90(1 \mathrm{H}, \mathrm{dd}, \mathrm{C} 5$, $\mathrm{J}=1.3 \mathrm{~Hz}$, and $15.4 \mathrm{~Hz}), 3.77(1 \mathrm{H}, \mathrm{d}, \mathrm{J}=15.6 \mathrm{~Hz}), 3.56(1 \mathrm{H}, \mathrm{d}, \mathrm{C} 5, \mathrm{~J}=15.0 \mathrm{~Hz}) ;{ }^{13} \mathrm{C}$ NMR: $171.72(\mathrm{C} 4)$, 139.24, 138.29, 138.22, 130.79, 130.71, 129.78, 129.60, 127.61, 124.37, 124.34 (ar), 63.22 (C2), 46.17, 33.33 (C5); (m/z) $288\left([\mathrm{M}+\mathrm{H}]^{+}\right) \mathrm{C}_{16} \mathrm{H}_{14} \mathrm{NOSF}(287.35)$.

3-(p-chlorobenzyl)-2-phenyl-1,3-thiazolidin-4-one (5d) (31\%); m.p. 156-157 ${ }^{\circ} \mathrm{C} ; \mathrm{cm}^{-1} 1667(\mathrm{C}=\mathrm{O}) ;{ }^{1} \mathrm{H}$ NMR $\left(\mathrm{CDCl}_{3}\right)$ 7.40-7.01 $(9 \mathrm{H}, \mathrm{m}$, aromatics), $5.36(1 \mathrm{H}, \mathrm{d}, \mathrm{C} 2, \mathrm{~J}=1.8 \mathrm{~Hz}), 5.07(1 \mathrm{H}, \mathrm{d}, \mathrm{J}=14.8 \mathrm{~Hz}), 3.90(1 \mathrm{H}, \mathrm{dd}, \mathrm{C} 5$, $\mathrm{J}=1.9 \mathrm{~Hz}$, and $14.4 \mathrm{~Hz}), 3.75(1 \mathrm{H}, \mathrm{d}, \mathrm{J}=15.6 \mathrm{~Hz}), 3.55(1 \mathrm{H}, \mathrm{d}, \mathrm{C} 5, \mathrm{~J}=14.8 \mathrm{~Hz}) ;{ }^{13} \mathrm{C}$ NMR: $171.70(\mathrm{C} 4)$, $139.23,134.26,134.24,130.24,129.77,129.60,129.35,127.60$ (ar), 63.18 (C2), 46.00, 33.39 (C5); (m/z) 303.5 $\left([\mathrm{M}+\mathrm{H}]^{+}\right) \mathrm{C}_{16} \mathrm{H}_{14} \mathrm{NOSCl}(303.81)$.

3-(m-chlorobenzyl)-2-phenyl-1,3-thiazolidin-4-one (5e) (38\%); m.p. 82-84 ${ }^{\circ} \mathrm{C} ; \mathrm{cm}^{-1} 1668(\mathrm{C}=\mathrm{O})$; ${ }^{1} \mathrm{H}$ NMR $\left(\mathrm{CDCl}_{3}\right)$ 7.40-6.97 (9H, m, aromatics), $5.39(1 \mathrm{H}, \mathrm{d}, \mathrm{C} 2, \mathrm{~J}=1.8 \mathrm{~Hz}), 5.06(1 \mathrm{H}, \mathrm{d}, \mathrm{J}=14.9 \mathrm{~Hz}), 3.91(1 \mathrm{H}, \mathrm{dd}, \mathrm{C}$, $\mathrm{J}=1.3 \mathrm{~Hz}$, and $15.6 \mathrm{~Hz}), 3.78(1 \mathrm{H}, \mathrm{d}, \mathrm{J}=15.7 \mathrm{~Hz}), 3.56(1 \mathrm{H}, \mathrm{d}, \mathrm{C} 5, \mathrm{~J}=14.9 \mathrm{~Hz}) ;{ }^{13} \mathrm{C}$ NMR: $171.71(\mathrm{C} 4)$, $139.19,137.81,135.02,130.46,129.81,129.60,128.85,128.58,127.63,126.94$ (ar), 63.26 (C2), 46.16, 33.29 (C5); (m/z) $303.5\left([\mathrm{M}+\mathrm{H}]^{+}\right) \mathrm{C}_{16} \mathrm{H}_{14} \mathrm{NOSCl}(303.81)$.

3-(m-bromobenzyl)-2-phenyl-1,3-thiazolidin-4-one (5f) (8\%); m.p. 82-84 ${ }^{\circ} \mathrm{C}$; $\mathrm{cm}^{-1} 1667(\mathrm{C}=\mathrm{O})$; ${ }^{1} \mathrm{H}$ NMR $\left(\mathrm{CDCl}_{3}\right)$ 7.42-7.01 (9H, m, aromatics), $5.39(1 \mathrm{H}, \mathrm{d}, \mathrm{C} 2, \mathrm{~J}=1.8 \mathrm{~Hz}), 5.05(1 \mathrm{H}, \mathrm{d}, \mathrm{J}=14.9 \mathrm{~Hz}), 3.91(1 \mathrm{H}, \mathrm{dd}, \mathrm{C}$, $\mathrm{J}=1.3 \mathrm{~Hz}$, and $16.9 \mathrm{~Hz}), 3.78(1 \mathrm{H}, \mathrm{d}, \mathrm{J}=15.6 \mathrm{~Hz}), 3.56(1 \mathrm{H}, \mathrm{d}, \mathrm{C} 5, \mathrm{~J}=14.9 \mathrm{~Hz}) ;{ }^{13} \mathrm{C}$ NMR: $171.27(\mathrm{C} 4)$, $138.72,137.62,131.34,131.08,130.30,129.39,129.17,127.20,127.00,122.76$ (ar), 62.84 (C2), 45.68, 32.90 (C5); (m/z) $349\left([\mathrm{M}+\mathrm{H}]^{+}\right) \mathrm{C}_{16} \mathrm{H}_{14} \mathrm{NOSBr}(348.26)$.

3-benzyl-2-phenyl-1,3-thiazolidin-4-one (5g) (51\%); m.p. 150-151 ${ }^{\circ} \mathrm{C} ; \mathrm{cm}^{-1} 1669(\mathrm{C}=\mathrm{O}) ;{ }^{1} \mathrm{H}$ NMR $\left(\mathrm{CDCl}_{3}\right)$ 7.40-7.09 (10H, m, aromatics), $5.39(1 \mathrm{H}, \mathrm{d}, \mathrm{C} 2, \mathrm{~J}=1.9 \mathrm{~Hz}), 5.17(1 \mathrm{H}, \mathrm{d}, \mathrm{J}=14.7 \mathrm{~Hz}), 3.91(1 \mathrm{H}, \mathrm{dd}, \mathrm{C} 5, \mathrm{~J}=1.9$ $\mathrm{Hz}$, and $14.4 \mathrm{~Hz}), 3.77(1 \mathrm{H}, \mathrm{d}, \mathrm{J}=15.6 \mathrm{~Hz}), 3.53(1 \mathrm{H}, \mathrm{d}, \mathrm{C} 5, \mathrm{~J}=14.6 \mathrm{~Hz}) ;{ }^{13} \mathrm{C}$ NMR: $171.66(\mathrm{C} 4), 139.56$, $135.71,129.64,129.56,129.20,128.85,128.36,127.58(\mathrm{ar}), 63.13(\mathrm{C} 2), 46.62,33.46(\mathrm{C} 5) ;(\mathrm{m} / \mathrm{z}) 270\left([\mathrm{M}+\mathrm{H}]^{+}\right)$ $\mathrm{C}_{16} \mathrm{H}_{15} \mathrm{NOS}$ (269.36).

3-(p-methylbenzyl)-2-phenyl-1,3-thiazolidin-4-one (5h) (50\%); m.p. 156-157 ${ }^{\circ} \mathrm{C} ; \mathrm{cm}^{-1} 1665(\mathrm{C}=\mathrm{O}) ;{ }^{1} \mathrm{H}$ NMR $\left(\mathrm{CDCl}_{3}\right)$ 7.39-6.97 (9H, m, aromatics), $5.37(1 \mathrm{H}, \mathrm{d}, \mathrm{C} 2, \mathrm{~J}=1.8 \mathrm{~Hz}), 5.14(1 \mathrm{H}, \mathrm{d}, \mathrm{J}=14.6 \mathrm{~Hz}), 3.92(1 \mathrm{H}, \mathrm{dd}, \mathrm{C}$, $\mathrm{J}=1.8 \mathrm{~Hz}$, and $15.6 \mathrm{~Hz}), 3.75(1 \mathrm{H}, \mathrm{d}, \mathrm{J}=15.5 \mathrm{~Hz}), 3.47(1 \mathrm{H}, \mathrm{d}, \mathrm{C} 5, \mathrm{~J}=14.6 \mathrm{~Hz}), 2.34\left(3 \mathrm{H}, \mathrm{s}, \mathrm{CH}_{3}\right) ;{ }^{13} \mathrm{C} \mathrm{NMR}$ : 171.59 (C4), 139.65, 138.11, 132.61, 129.85, 129.59, 129.54, 128.86, 127.56 (ar), 63.04 (C2), 46.31, 33.52 (C5), $21.66\left(\mathrm{CH}_{3}\right) ;(\mathrm{m} / \mathrm{z}) 284\left([\mathrm{M}+\mathrm{H}]^{+}\right) \mathrm{C}_{17} \mathrm{H}_{17} \mathrm{NOS}(283.39)$.

3-(m-methylbenzyl)-2-phenyl-1,3-thiazolidin-4-one (5i) (1\%); m.p. 80-82 ${ }^{\circ} \mathrm{C} ; \mathrm{cm}^{-1} 1671(\mathrm{C}=\mathrm{O}) ;{ }^{1} \mathrm{H}$ NMR $\left(\mathrm{CDCl}_{3}\right)$ 7.42-6.87 $(9 \mathrm{H}, \mathrm{m}$, aromatics), $5.39(1 \mathrm{H}, \mathrm{d}, \mathrm{C} 2, \mathrm{~J}=1.8 \mathrm{~Hz}), 5.13(1 \mathrm{H}, \mathrm{d}, \mathrm{J}=14.7 \mathrm{~Hz}), 3.92(1 \mathrm{H}, \mathrm{dd}, \mathrm{C} 5$, $\mathrm{J}=1.9 \mathrm{~Hz}$, and $15.6 \mathrm{~Hz}), 3.77(1 \mathrm{H}, \mathrm{d}, \mathrm{J}=15.6 \mathrm{~Hz}), 3.48(1 \mathrm{H}, \mathrm{d}, \mathrm{C} 5, \mathrm{~J}=14.6 \mathrm{~Hz}), 2.32\left(3 \mathrm{H}, \mathrm{s}, \mathrm{CH}_{3}\right) ;{ }^{13} \mathrm{C} \mathrm{NMR}$ : 171.62 (C4), 139.66, 138.96, 135.57, 129.60, 129.53, 129.52, 129.10, 129.00, 127.55, 125.90 (ar), 63.13 (C2), 46.58, 33.48 (C5), $21.80\left(\mathrm{CH}_{3}\right) ;(\mathrm{m} / \mathrm{z}) 284\left([\mathrm{M}+\mathrm{H}]^{+}\right) \mathrm{C}_{17} \mathrm{H}_{17} \mathrm{NOS}(283.39)$.

3-(p-methoxybenzyl)-2-phenyl-1,3-thiazolidin-4-one (5j) (62\%); m.p. 147-148 ${ }^{\circ} \mathrm{C}$; $\mathrm{cm}^{-1} 1663(\mathrm{C}=\mathrm{O})$; ${ }^{1} \mathrm{H}$ NMR $\left(\mathrm{CDCl}_{3}\right) 7.39-6.81(9 \mathrm{H}, \mathrm{m}$, aromatics), $5.37(1 \mathrm{H}, \mathrm{d}, \mathrm{C} 2, \mathrm{~J}=1.8 \mathrm{~Hz}), 5.10(1 \mathrm{H}, \mathrm{d}, \mathrm{J}=14.6 \mathrm{~Hz}), 3.90(1 \mathrm{H}, \mathrm{dd}, \mathrm{C} 5$, $\mathrm{J}=1.3 \mathrm{~Hz}$, and $15.6 \mathrm{~Hz}), 3.79\left(3 \mathrm{H}, \mathrm{s}, \mathrm{CH}_{3}\right), 3.73(1 \mathrm{H}, \mathrm{d}, \mathrm{J}=15.6 \mathrm{~Hz}), 3.47(1 \mathrm{H}, \mathrm{d}, \mathrm{C} 5, \mathrm{~J}=14.6 \mathrm{~Hz}) ;{ }^{13} \mathrm{C} \mathrm{NMR}$ : 170.73 (C4), 158.87, 128.82, 129.45, 128.77, 128.72, 126.92, 126.76, 113.68 (ar), 62.23 (C2), $54.87\left(\mathrm{CH}_{3}\right)$, 45.21, 33.74 (C5); (m/z) $301\left([\mathrm{M}+\mathrm{H}]^{+}\right) \mathrm{C}_{17} \mathrm{H}_{17} \mathrm{NO}_{2} \mathrm{~S}(299.39)$.

3-(m-methoxybenzyl)-2-phenyl-1,3-thiazolidin-4-one (5k) (2\%); m.p. 74-75 ${ }^{\circ} \mathrm{C} ; \mathrm{cm}^{-1} 1664(\mathrm{C}=\mathrm{O})$; ${ }^{1} \mathrm{H}$ NMR $\left(\mathrm{CDCl}_{3}\right)$ 7.39-6.63 (9H, m, aromatics), $5.40(1 \mathrm{H}, \mathrm{d}, \mathrm{C} 2, \mathrm{~J}=1.4 \mathrm{~Hz}), 5.14(1 \mathrm{H}, \mathrm{d}, \mathrm{J}=14.7 \mathrm{~Hz}), 3.91(1 \mathrm{H}, \mathrm{dd}, \mathrm{C} 5$, $\mathrm{J}=1.2 \mathrm{~Hz}$, and $15.8 \mathrm{~Hz}), 3.77\left(3 \mathrm{H}, \mathrm{s}, \mathrm{CH}_{3}\right), 3.76(1 \mathrm{H}, \mathrm{d}, \mathrm{J}=15.6 \mathrm{~Hz}), 3.50(1 \mathrm{H}, \mathrm{d}, \mathrm{C} 5, \mathrm{~J}=14.8 \mathrm{~Hz}) ;{ }^{13} \mathrm{C} \mathrm{NMR}$ : 171.63 (C4), 160.28, 139.58, 137.21, 130.22, 129.63, 129.54, 127.59, 121.07, 114.30, 113.81 (ar), 63.12 (C2), $55.65\left(\mathrm{CH}_{3}\right), 46.57,33.43(\mathrm{C} 5) ;(\mathrm{m} / \mathrm{z}) 301\left([\mathrm{M}+\mathrm{H}]^{+}\right) \mathrm{C}_{17} \mathrm{H}_{17} \mathrm{NO}_{2} \mathrm{~S}(299.39)$.

\section{Acknowledgements}

The work described here was completed by Daniel McGarity in partial fulfilment of his Schreyer Honors Thesis and Bachelor of Science degree at the Pennsylvania State in Spring 2008. We would like to acknowledge the 
Pennsylvania State University Schreyer Honors College for funding this work.

\section{References}

Barreca, M. L., Chimirri, A., De Luca, L., Monforte, A.,Monforte, P., Rao, A., ... Witvrouw, M. (2001). Discovery of 2,3-diaryl-1,3 thiazolidin-4-ones as potent anti-HIV-1 agents. Bioorganic \& Medicinal Chemistry Letters, 11, 1793-1796. http://dx.doi.org/10.1016/S0960-894X(01)00304-3

Cannon, K., Mascavage, L., Kistler, K., Tierney, J., Yennawar, H., \& Lagalante, A. (2013). An Experimental and Theoretical Conformational Study of a Series of Substituted 3-cyclohexyl-2-phenyl-1,3-thiazolidin-4-ones. International Journal of Chemistry, 5, 46-56. http://dx.doi.org/10.5539/ijc.v5n3p46

Fun, H.-K., Hemamalini, M., Shanmugavelan, P., Ponnuswamy, A., \& Jagatheesan, R. (2011). 3-Benzyl-2-phenyl-1,3-thiazolidin-4-one. Acta Cryst. http://dx.doi.org/10.1107/S1600536811037706

Hafez, N. H., \& Gazzar, A. B. A. (2009). ynthesis and antitumor activity of substituted triazolo[4,3-a]pyrimidin-6-sulfonamide with an incorporated thiazolidinone moiety. Bioorganic \& Medicinal Chemistry Letters, 19(15), 4143-4147. http://dx.doi.org/10.1016/j.bmcl.2009.05.126

Ravichandran, V. Prashantha Kumar, B. R., Sankar, S., \& Agrawal, R. K. (2009). Predicting anti-HIV activity of 1,3,4-thiazolidinone derivatives: 3D-QSAR approach. European Journal of Medicinal Chemistry, 44(3), 1180-1187. http://dx.doi.org/10.1016/j.ejmech.2008.05.036

Silverberg, L. J., Bear, E. R., Foose, K., Kirkland, K., McElvaney, R., Cannon, K., ... Lagalante, A. (2013) Verifying the Predict ability of ${ }^{13} \mathrm{C}$ Chemical Shifts for a Series of Substituted-2-(4-Chlorophenyl)-3-Phenyl-1,3-thiazolidin-4-ones. International Journal of Chemistry, 5(4), 120-127. http://dx.doi.org/10.5539/ijc.v5n4p120

Surrey, A. R. (1947). The Preparation of 4-Thiazolidones by the reaction of thioglycolic acid with Schiff Bases. Journal of the American Chemical Society, 69(11), 2911. http://dx.doi.org/10.1021/ja01203a507

Tierney, J., Houghton, G., Sanford, K., Mascavage, L., McCoy, M., Findeisen, A., \& Kilburn, J. (1996a). Effects and conformational analysis of some substituted 2,3-diphenyl-1,3-thiazolidin-4-ones. Magnetic Resonance in Chemistry, 34, 573-576. http://dx.doi.org/10.1002/(SICI)1097-458X(199608)34:8<573::AID-OMR928>3.0.CO;2-D

Tierney, J., Sheridan, D., Kovalesky, K., Mascavage, L. M., Schneider, K., \& Sheely, C. (1996b). Substituent effects for some substituted 3-benzyl-2-phenyl-1,3-thiazolidin-4-ones using ${ }^{1} \mathrm{H}$ and ${ }^{13} \mathrm{C}$ NMR. Heterocyclic Communications, 6, 105-111. http://dx.doi.org/10.1515/HC.2000.6.2.105

Vigorita, M. G., Ottana, R., Monforte, F., Maccan, R., Trovatto, A., Monforte, M. T., \& Taviano, M. F. (2001). Syn thesis and antiinflammatory, analgesic activity of 3,3'-(1,2-ethanediyl)-bis[2-aryl-4-thiazolidinone] chiral compounds. Part 10. Bioorganic \& Medicinal Chemistry Letters, 11(21), 2791-2794. http://dx.doi.org/10.1016/S0960-894X(01)00476-0

Woolston, C. R. J., Lee, J. B., Swinbourne, F. J., \& Thomas, W. A. (1992). Proton NMR investigation of some substituted 1,3-thiazolidin-4-ones. Magnetic Resonance in Chemistry, 30, 1075-1078. http://dx.doi.org/10.1002/mrc.1260301109

Yennawar, H. P., Tierney, J., \& Silverberg, L. J. (2014). 2,3-Diphenyl-1,3-thiazolidin-4-one. Acta Crystallographica, Section E70, o847. http://dx.doi.org/10.1107/S1600536814015128

\section{Copyrights}

Copyright for this article is retained by the author(s), with first publication rights granted to the journal.

This is an open-access article distributed under the terms and conditions of the Creative Commons Attribution license (http://creativecommons.org/licenses/by/3.0/). 